\title{
Labial salivary gland biopsy and secondary Sjögren's syndrome: where we are and where we want to be
}

\author{
Ciro Manzo \\ Internal and Geriatric Medicine Department, Rheumatologic Outpatient Clinic Hospital „Mariano Lauro”, Sant’Agnello, Italy
}

\section{Dear Editor,}

I read with great interest the paper recently published by Sebastian et al., in which the authors summarized the previously used and current classification criteria for secondary Sjogren's syndrome (sSS), and underlined "the need for labial salivary gland biopsy in all cases in which we suspect sSS to confirm the diagnosis" [1].

As is well known, labial salivary gland (LSG) biopsy is anything but easy and straightforward. Vivino et al. [2] reported that a second expert evaluation of 58 LSGS re-analyzed by a single center led to revision of the initial diagnosis in $53 \%$ of the patients. More recently, Costa et al. [3] reported a multicenter cohort study in which LSG biopsies were analyzed with a standard blinded assessment by two different pathologists at a 2-month interval. The analysis included the measurement of focus score (FS) and detection of germinal center (GC)-like structures. The inter-observer variability comparison revealed poor agreement for the detection and calculation of FS and detection of focal lymphocytic sialadenitis (FLS), lack of concordance for the presence of duct dilation and (less for) fibrosis. In more than $12 \%$ of the cases, the second evaluation by trained pathologists led to a diagnosis change [3].

In older patients, the presence of age-related findings may generate further confusion. For example, some

Table I. Tarpley's grading system for LSG biopsy [modified based on 9]

\begin{tabular}{|lr|}
\hline Grade & Description of gland tissue \\
\hline 0 & Normal \\
\hline 1 & 1 or 2 aggregates \\
\hline 2 & $>3$ aggregates \\
\hline 3 & $\begin{array}{r}\text { Diffuse infiltrate with partial destruction of acinar } \\
\text { tissue, with or without fibrosis }\end{array}$ \\
\hline 4 & $\begin{array}{l}\text { Diffuse infiltrate (with or without fibrosis) } \\
\text { destroying the entire lobular architecture }\end{array}$ \\
\hline
\end{tabular}

investigators found that acinar atrophy and fibrosis are common in healthy individuals aged over 65 years, FS may be higher in older age groups, and the increased area of fat tissue may be a selective feature of aging [4-6].

More recently, the Sjögren's histopathology workshop performed by the EULAR Sjögren's Syndrome Experimental and Translational Investigative Alliance (ESSENTIAL) study group provided a consensus guidance for the use of LSG histopathology in clinical trials. The diagnostic importance of foci that are adjacent to normal parenchyma was emphasized and several recommendations were proposed. In particular, recommendation number 6 suggested that the extent of the atrophic features should be graded as mild, moderate, and severe, in addition to the presence or absence of FLS. Instead, recommendation number 10 underlined the necessity that all foci should be included in the FS and in foci calculations, even when adjacent to abnormal acini or ducts. However, the level of these recommendations was low [7].

As for today, there is still a strong need to achieve a consensus among experts on how to differentiate in LSG biopsy the Sjogren's typical findings from the age-related ones [8]. The use of a grading score taking the destruction of acinar tissue and fibrosis into account could help (Table I).

Furthermore, as recently highlighted by Mavragani and Moutsopoulos [10], immunohistochemical studies comparing the composition of lymphocytic infiltrates in LSG biopsy revealed differences almost exclusively in rheumatoid arthritis associated with sicca-related manifestations, and not in other systemic autoimmune rheumatic diseases associated with Sjögren's syndrome.

Therefore, is LSG biopsy crucial to confirm the diagnosis of sSS? Not always. It often becomes itself an element of discussion or confusion.

The author declares no conflict of interest.

Address for correspondence:

Ciro Manzo, Internal and Geriatric Medicine Department ASL NA sud, Rheumatologic Outpatient Clinic Hospital „Mariano Lauro”, viale dei Pini 1, 80065 Sant'Agnello, Italy, e-mail: manzoreumatologo@libero.it

Submitted: 26.11.2019; Accepted: 28.11.2019 


\section{References}

1. Sebastian A, Szachowicz A, Wiland P. Classification criteria for secondary Sjögren's syndrome. Current state of knowledge. Reumatologia 2019; 57: 277-280.

2. Vivino FB, Gala I, Hermann GA. Change in final diagnosis on second evaluation of labial minor salivary gland biopsies. J Rheumatol 2002; 29: 938-944.

3. Costa S, Quintin-Roué I, Lesourd A, et al. Reliability of histopathological salivary gland biopsy assessment in Sjögren's syndrome: a multicenter cohort study. Rheumatology (Oxford) 2015; 54: 1056-1064.

4. Radfar L, Kleiner DE, Fox PC, Pillemer SR. Prevalence and clinical significance of lymphocytic foci in minor salivary glands of health volunteers. Arthritis Rheum 2002; 47: 520-524.

5. Vered M, Buchner A, Boldon P, Dayan D. Age-related histomorphometric changes in labial salivary glands with special reference to the acinar component. Exp Gerontol 2000; 35: 1075-1084.
6. Leehan KM, Pezant NP, Rasmussen A, et al. Fatty infiltration of the minor salivary glands is a selective feature of aging but not Sjögren's syndrome. Autoimmunity 2017; 50: 451-457.

7. Fisher BA, Jonsson R, Daniels T, et al. Standardization of labial salivary gland histopathology in clinical trials in primary Sjögren's syndrome. Ann Rheum Dis 2017; 76: 1161-1168.

8. Manzo C, Maslinska M. Primary Sjögren's syndrome in the elderly: does age of onset make a difference? EMJ Rheumatol 2018; 5: 75-82

9. Tarpley TM Jr, Anderson LG, White CL. Minor salivary gland involvement in Sjögren's syndrome. Oral Surg Oral Med Oral Pathol 1974; 37: 64-74.

10. Mavragani CP, Moutsopoulos HM. Primary versus secondary Sjögren syndrome: is it time to reconsider these terms? J Rheumatol 2019; 46: 665-666. 